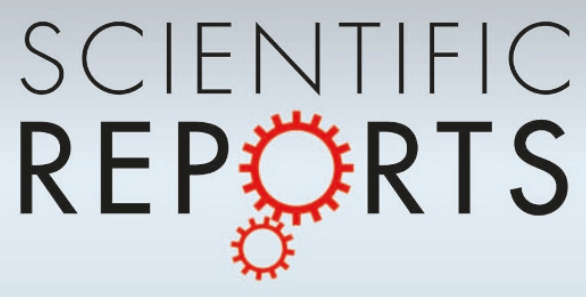

\title{
OPEN A universal quantum information \\ QUANTUM processor for scalable quantum
communication and networks
}

SUBJECT AREAS:

INFORMATION

OPTICAL MANIPULATION AND TWEEZERS

Received

28 July 2014

Accepted

29 September 2014

Published

15 October 2014

Correspondence and requests for materials should be addressed to

X.Y. lyangxih $1 @$

yahoo.com) or S.Z.

(syzhu@csrc.ac.cn)

\author{
Xihua Yang', Bolin Xue', Junxiang Zhang ${ }^{2} \&$ Shiyao Zhu ${ }^{3}$
}

\begin{abstract}
'Department of Physics, Shanghai University, Shanghai 200444, China, ${ }^{2}$ State Key Laboratory of Quantum Optics and Quantum Optics Devices, Institute of Opto-Electronics, Shanxi University, Taiyuan 030006, China, ${ }^{3}$ Beijing Computational Science Research Center, Beijing 100084, China.
\end{abstract}

Entanglement provides an essential resource for quantum computation, quantum communication, and quantum networks. How to conveniently and efficiently realize the generation, distribution, storage, retrieval, and control of multipartite entanglement is the basic requirement for realistic quantum information processing. Here, we present a theoretical proposal to efficiently and conveniently achieve a universal quantum information processor (QIP) via atomic coherence in an atomic ensemble. The atomic coherence, produced through electromagnetically induced transparency (EIT) in the $\Lambda$-type configuration, acts as the QIP and has full functions of quantum beam splitter, quantum frequency converter, quantum entangler, and quantum repeater. By employing EIT-based nondegenerate four-wave mixing processes, the generation, exchange, distribution, and manipulation of light-light, atom-light, and atom-atom multipartite entanglement can be efficiently and flexibly achieved in a deterministic way with only coherent light fields. This method greatly facilitates the operations in quantum information processing, and holds promising applications in realistic scalable quantum communication and quantum networks.

T he generation of light-light, matter-matter, and light-matter multipartite entanglement plays an essential role in quantum communication and quantum networks ${ }^{1-3}$. One commonly-used method to create multiple entangled fields is to combine squeezed fields produced through parametric down-conversion processes in nonlinear optical crystals with linear beam splitters or polarizing beam splitters; however, the created entangled fields are degenerate and suffer from short correlation time. At the nodes of quantum networks, multiple entangled fields with different frequencies are necessary to connect different physical systems. The creation of nondegenerate multipartite entanglement has been investigated by using nonlinear optical processes. Nussenzveig et al. demonstrated the production of pump-signal-idler three-color entanglement in the abovethreshold optical parametric oscillator $(\mathrm{OPO})^{4,5}$, and a scheme based on this three-color entanglement was proposed to generate scalable multipartite entanglement by using many OPOs operated in a chain configuration ${ }^{6}$; other ways have also been proposed by employing either cascaded nonlinearities or concurrent parametric oscillation $^{7,8}$. Nevertheless, these schemes are still of limited use due to relatively short correlation time. An alternative attractive avenue is the implementation of the electromagnetically induced transparency ${ }^{9-11}$ (EIT)based multi- $\Lambda$-type systems in an atomic ensemble. By using nondegenerate four-wave mixing (FWM) or Raman scattering processes, the generation of nondegenerate narrow-band multi-entangled fields with long correlation time have been actively studied ${ }^{12-20}$; in addition, the entangled state exchange between an atomic ensemble and light fields ${ }^{12-14}$, as well as between two atomic ensembles ${ }^{21-26}$, have also been realized, which are quite suitable for quantum memory and quantum networks. However, in these EIT-based schemes, the input laser fields for generating entanglement are treated classically and their quantum features are not explored.

In quantum communication and quantum networks, the distribution of quantum states over long distance is limited by inevitable photon loss in the transmission channel. To overcome this limitation, Briegel et al. ${ }^{27}$ introduced the concept of quantum repeater, combining entanglement swapping and quantum memory to extend communication distances. One conventionally-employed strategy for realizing quantum repeater is to use atomic ensembles as quantum memories, combining with linear optical techniques and photon counting to perform all required operations ${ }^{24-26}$. However, in such protocols, nonclassical input light fields and interferometric stabilization over long distance are required for entanglement swapping; moreover, the entanglement is created in a post-selection way. 
Motivated by the experimental observation of generating multifield correlations and anti-correlations via atomic spin coherence in ${ }^{85} \mathrm{Rb}$ atomic system ${ }^{28}$, here, we propose a convenient and efficient scheme to create a universal quantum information processor via atomic coherence in the multi- $\Lambda$-type atomic system. The atomic coherence, produced through electromagnetically induced transparency in the $\Lambda$-type configuration, acts as a quantum information processor and has the function of quantum beam splitter, quantum frequency converter, quantum entangler, and quantum repeater. By using the QIP, the generation, exchange, distribution, and manipulation of light-light, light-atom, as well as atom-atom multipartite entangled state, in principle, can be conveniently and flexibly achieved in a deterministic way with only coherent light fields. This method would greatly simplify the practical implementation, and may find potential applications in quantum communication and quantum networks.

\section{Results}

Mechanism of excitations and atom-light evolution equations. The considered model, as shown in Fig. 1a, is based on the experimental configuration in Ref. [28], where the relevant energy levels and applied/generated laser fields form a triple- $\Lambda$-type system. Levels $|1\rangle,|2\rangle$, and $|3\rangle$ correspond, respectively, to the ground-state hyperfine levels $5 \mathrm{~S}_{1 / 2}(\mathrm{~F}=3), 5 \mathrm{~S}_{1 / 2}(\mathrm{~F}=2)$, and the excited state $5 \mathrm{P}_{1 / 2}$ in $\mathrm{D}_{1}$ line of ${ }^{85} \mathrm{Rb}$ atom with the ground-state hyperfine splitting of $3.036 \mathrm{GHz}$. The probe field $\mathrm{E}_{\mathrm{p}}$ (with frequency $\omega_{p}$ and Rabi frequency $\Omega_{p}$ ) and coupling field $\mathrm{E}_{\mathrm{c}}$ (with frequency $\omega_{c}$ and Rabi frequency $\Omega_{c}$ ) are relatively strong and tuned to resonance with the transitions $|2\rangle-|3\rangle$ and $|1\rangle-|3\rangle$, respectively. By applying a third mixing field $\mathrm{E}_{\mathrm{m} 1}$ (or $\mathrm{E}_{\mathrm{m} 2}$ ) with frequency $\omega_{m 1}$ (or $\omega_{m 2}$ ), offresonantly coupling levels $|2\rangle$ (or $|1\rangle$ ) and $|3\rangle$ with detuning $\Delta_{1}=$ $\omega_{\mathrm{m} 1}-\omega_{32}$ (or $\Delta_{2}=\omega_{\mathrm{m} 2}-\omega_{31}$ ), a Stokes field $\mathrm{E}_{1}$ (or an anti-Stokes field $\mathrm{E}_{2}$ ) can be created through the nondegenerate FWM process. In fact, as shown in Fig. 1b, the produced Stokes field $E_{1}$ (or anti-Stokes field $\mathrm{E}_{2}$ ) can be equivalently regarded as scattering the field $\mathrm{E}_{\mathrm{m} 1}$ (or $\mathrm{E}_{\mathrm{m} 2}$ ) off the atomic coherence $\sigma_{12}$ pre-established by the strong coupling and probe fields in the $\Lambda$-type EIT configuration formed by levels $|1\rangle,|2\rangle$, and $|3\rangle$. In Refs. [18-20], we have employed the similar scheme to generate arbitrary number of nondegenerate narrow-band entangled fields and create quantum entangler via the pre-established atomic spin wave, however, the quantum properties of the scattering fields as well as the entangled feature between the scattering fields and the generated fields have not been investigated. In fact, as shown in Ref. [28], correlations and anti-correlations between the scattering and generated fields have been experimentally observed via atomic spin coherence in ${ }^{85} \mathrm{Rb}$ atomic system. In what follows, by using the Heisenberg-Langevin method with the scattering fields and the generated Stokes/antiStokes fields treated quantum mechanically, we show how the atomic coherence can act as a QIP and can be used to realize the exchange and distribution of multipartite entangled state.

By using the equivalent configuration shown in Fig. 1b, we first investigate the entangled feature among the scattering filed $\mathrm{E}_{\mathrm{m} 1}$, generated Stokes field $\mathrm{E}_{1}$, as well as the atomic coherence $\sigma_{12}$. We assume that the detunings $\Delta_{1}$ and $\Delta_{2}$ of the scattering fields are sufficiently large so that the coupling between different scattering fields can be neglected. Also, we assume that the coupling and probe fields for precreating the atomic coherence are substantially strong, so that the atomic coherence is strong enough to ensure that different scattering fields have negligible influence on it. Under these conditions, the laser-field interaction Hamiltonian in the rotating wave approximation has the form ${ }^{29-31}$

$$
\begin{aligned}
& \hat{V}=-\frac{\hbar N}{L} \int_{0}^{L} d z \\
& \left(\Delta_{1} \sigma_{33}(z, t)+g_{1} a_{1}(z, t) \sigma_{31}(z, t)+g_{2} b_{1}(z, t) \sigma_{32}(z, t)+H . c .\right),
\end{aligned}
$$

where $\sigma_{31}(z, t), \sigma_{32}(z, t)$, and $\sigma_{33}(z, t)$ are the collective atomic operators, $a_{1}(z, t)$ and $b_{1}(z, t)$ are the quantum operators of the generated Stokes field $\mathrm{E}_{1}$ and scattering field $\mathrm{E}_{\mathrm{m} 1}, g_{1(2)}=\mu_{13(23)} \cdot \varepsilon_{1(2)} / \hbar$ is the atom-field coupling constant with $\mu_{13(23)}$ as the dipole moment for the 1-3 (2-3) transition and $\varepsilon_{1(2)}=\sqrt{\hbar \omega_{1(2)} / 2 \epsilon_{0} V}$ as the electric field of a single Stokes (scattering) photon with $\epsilon_{0}$ as the free space permittivity and $\mathrm{V}$ as the interaction volume with length $\mathrm{L}$ and beam radius $\mathrm{r}$, and $\mathrm{N}$ is the total number of atoms in the quantum volume. The HeisenbergLangevin equations for describing the evolution of the atomic operators $\sigma_{12}(z, t), \sigma_{13}(z, t)$, and $\sigma_{23}(z, t)$ can be written as ${ }^{29-31}$

${ }^{85} \mathrm{Rb} \mathrm{D}_{1}$-line

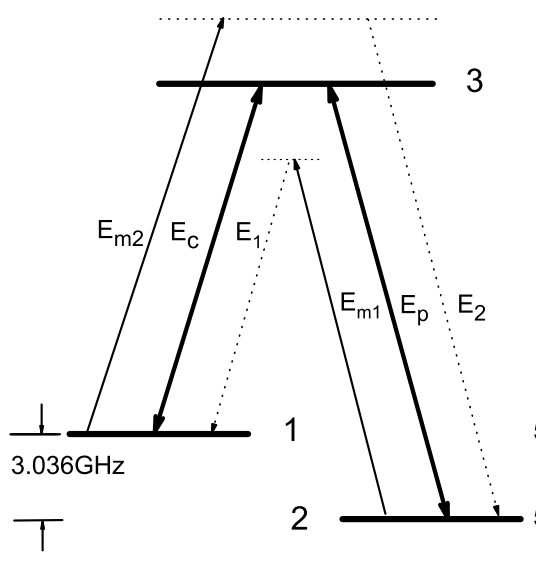

(a)

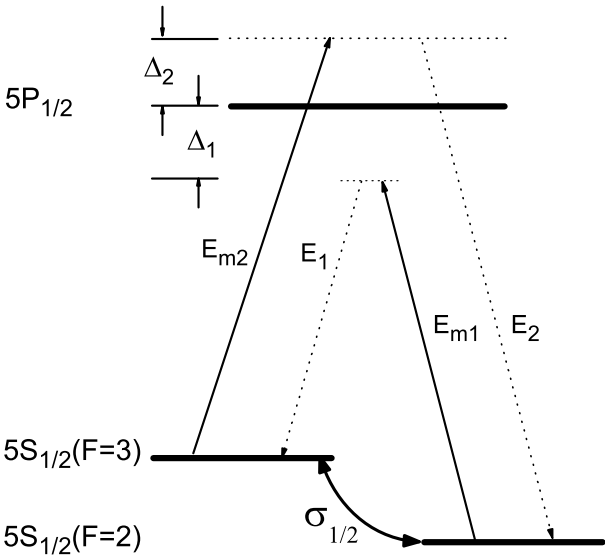

(b)

Figure $1 \mid$ (a) The triple- $\Lambda$-type system of the $\mathrm{D}_{1}$ transitions in ${ }^{85} \mathrm{Rb}$ atom coupled by the coupling $\left(\mathrm{E}_{\mathrm{c}}\right)$, probe $\left(\mathrm{E}_{\mathrm{p}}\right)$, and mixing $\left(\mathrm{E}_{\mathrm{m} 1}\right.$ and $\left.\mathrm{E}_{\mathrm{m} 2}\right)$ fields based on the experimental configuration used in Ref. [28], where $E_{p}$ and $E_{c}$ fields both resonantly drive $|2\rangle-|3\rangle$ and $|1\rangle-|3\rangle$ transitions, and the corresponding Stokes fields $\mathrm{E}_{1}$, and anti-Stokes fields $\mathrm{E}_{2}$ are generated through two FWM processes. (b) The equivalent configuration of (a) with the two lower states driven by the atomic coherence $\sigma_{12}$ precreated by the strong on-resonant $\mathrm{E}_{\mathrm{c}}$ and $\mathrm{E}_{\mathrm{p}}$ fields in the $\Lambda$-type EIT configuration. 


$$
\begin{gathered}
\dot{\sigma}_{12}(z, t)=-\gamma_{0} \sigma_{12}-i g_{1} a_{1}(z, t) \sigma_{23}^{+}+i g_{2} b_{1}^{+}(z, t) \sigma_{13}+F_{12}(z, t), \\
\dot{\sigma}_{13}(z, t)=-\left[\gamma_{13}-i \Delta_{1}\right] \sigma_{13}+i g_{1} a_{1}(z, t)\left(\sigma_{11}-\sigma_{33}\right) \\
+i g_{2} b_{1} \sigma_{12}+F_{13}(z, t), \\
\dot{\sigma}_{23}(z, t)=-\left(\gamma_{23}-i \Delta_{1}\right) \sigma_{23}+ \\
i g_{2} b_{1}(z, t)\left(\sigma_{22}-\sigma_{33}\right)+i g_{1} a_{1} \sigma_{12}^{+}+F_{23}(z, t),
\end{gathered}
$$

where $\gamma_{13}=\gamma_{23}=\frac{\gamma_{1}+\gamma_{2}}{2}$ with $\gamma_{1}$ and $\gamma_{2}$ being the population decay rates from level 3 to levels 1 and $2, \gamma_{0}$ is the coherence decay rate between levels 1 and 2 , and $F_{i j}(z, t)$ are the collective atomic $\delta$-correlated Langevin noise operators. The noise correlation function for the noise operators $F_{i j}(z, t)$ can be expressed as $\left\langle F_{i j}(z, t) F_{i^{\prime} j^{\prime}}\left(z^{\prime}, t^{\prime}\right)\right\rangle=$ $\frac{L}{N} \mathcal{D}_{i j, i^{\prime} j^{\prime}}(z, t) \delta\left(z-z^{\prime}\right) \delta\left(t-t^{\prime}\right)$, where $\mathcal{D}_{i j, i^{\prime} j^{\prime}}(z, t)$ is the Langevin diffusion coefficient, which can be calculated by using the generalized dissipation-fluctuation theorem ${ }^{29}$. In the similar analysis described in Refs. [29-31], under the assumption that the uniformly-distributed pencil-shaped atomic sample is optically thin in the transverse direction, the evolution of the annihilation operators $a_{1}$ and $b_{1}$ can be described by the coupled propagation equations

$$
\begin{aligned}
& \left(\frac{\partial}{\partial t}+c \frac{\partial}{\partial z}\right) a_{1}(z, t)=i g_{1} N \sigma_{13}, \\
& \left(\frac{\partial}{\partial t}+c \frac{\partial}{\partial z}\right) b_{1}(z, t)=i g_{2} N \sigma_{23} .
\end{aligned}
$$

We use the perturbation analysis to treat the interaction of the atoms with the fields. In the zeroth-order perturbation expansion, by semiclassically treating the interaction of the atoms with the strong coupling and probe fields in the $\Lambda$-type EIT configuration, we get the steady-state mean values of the atomic operators $\sigma_{11}^{(0)}, \sigma_{22}^{(0)}, \sigma_{33}^{(0)}$, $\sigma_{12}^{(0)}, \sigma_{13}^{(0)}$ and $\sigma_{23}^{(0)}$. By substituting the zeroth-order solution into the Fourier-transformed Heisenberg-Langevin equations for the atomic operators $\sigma_{12}(z, t), \sigma_{13}(z, t)$, and $\sigma_{23}(z, t)$, we can get the first-order solution $\sigma_{12}^{(1)}(z, \omega), \sigma_{13}^{(1)}(z, \omega)$, and $\sigma_{23}^{(1)}(z, \omega)$, which are expressed as

$$
\sigma_{12}^{(1)}(z, \omega)=\frac{1}{\gamma_{0}+i \omega}\left[-i g_{1} \sigma_{23}^{+(0)} a_{1}+i g_{2} \sigma_{13}^{(0)} b_{1}^{+}+F_{12}\right],
$$

$\sigma_{13}^{(1)}(z, \omega)=\frac{1}{\gamma_{13}+i\left(\omega-\Delta_{1}\right)}\left[i g_{1}\left(\sigma_{11}^{(0)}-\sigma_{33}^{(0)}\right) a_{1}+i g_{2} \sigma_{12}^{(0)} b_{1}+F_{13}\right]$,

$\sigma_{23}^{(1)}(z, \omega)=\frac{1}{\gamma_{23}+i\left(\omega-\Delta_{1}\right)}\left[i g_{2}\left(\sigma_{22}^{(0)}-\sigma_{33}^{(0)}\right) b_{1}+i g_{1} \sigma_{12}^{+(0)} a_{1}+F_{23}\right]$,

Note that in the above first-order analysis, we only consider the steady-state mean values of the zeroth-order atomic operators (neglecting the noise operators as done in Refs. [29-31]) for the first-order solution. Substituting $\sigma_{13}^{(1)}$ and $\sigma_{23}^{(1)}$ into Fourier-transformed coupled propagation equations, the output of the annihilation operators $a_{1}(L, \omega)$ and $b_{1}(L, \omega)$ for the Stokes and scattering fields with respect to the Fourier frequency $\omega$ can be obtained, which is a linear combination of the input of the operators $a_{1}(0, \omega)$ and $b_{1}(0, \omega)$ and Langevin noise terms, having the analogous expression in Refs. [29-31].

Generation and distribution of multipartite entanglement. To test the entangled feature between the scattering field and Stokes field, as well as the atomic coherence $\sigma_{12}$, we use the criterion $\mathrm{V}_{\mathrm{ij}}=(\Delta \mu)^{2}+$
$(\Delta v)^{2}<4$ proposed in Ref. [32], where $\mu=x_{i} \pm x_{j}$ and $v=p_{i} \mp p_{j}$ ("+" in $\mu$ and "- " in $v$ for both $\mathrm{V}_{\mathrm{a} 1-\mathrm{b} 1}$ and $\mathrm{V} \sigma_{12 \text {-b1 }}$, whereas " -" in $\mu$ and " + " in $v$ for $\left.\mathrm{V}_{\mathrm{a} 1-} \sigma_{12}\right)$ with $x_{i}=\left(a_{i}+a_{i}^{+}\right)$and $p_{i}=-i\left(a_{i}-a_{i}^{+}\right)$. Satisfying the above inequality is sufficient to demonstrate the creation of bipartite entanglement, and the smaller the correlation $\mathrm{V}$ is, the stronger the degree of the bipartite entanglement becomes. As shown in Fig. 1, the Stokes field is initially in vacuum, and the scattering field is assumed to be initially in a coherent state $|\alpha\rangle$. In the following, the relevant parameters are scaled with $\mathrm{m}$ and $\mathrm{MHz}$, or $\mathrm{m}^{-1}$ and $\mathrm{MHz}^{-1}$, and set according to the experimental conditions in Ref. [28] with atomic density $n_{0}=5 \times 10^{19}, r=1 \times 10^{-4}, L=0.06, \gamma_{1}$ $=\gamma_{2}=3, \gamma_{0}=0.1, \omega_{12}=3036, \Omega_{p}=\Omega_{c}=400, \Delta_{1}=-1000$, and $\Delta_{2}$ $=1000$.

Figure 2 shows the evolution of correlations $\mathrm{V}_{\mathrm{a} 1-\mathrm{b} 1}, \mathrm{~V}_{\mathrm{a} 1-} \sigma_{12}$, and $\mathrm{V} \sigma_{12 \text {-b1 }}$ as a function of the Fourier frequency $\omega$. It can be seen that, nearly in the whole range of the Fourier frequency $\omega, \mathrm{V}_{\mathrm{a} 1-\mathrm{b} 1}, \mathrm{~V}_{\mathrm{a} 1-} \sigma_{12}$, and $\mathrm{V} \sigma_{12-\mathrm{b} 1}$ are always less than 4 , which sufficiently demonstrates that the scattering field, Stokes field, and atomic coherence are genuinely entangled with each other. In a wide range $(-2600 \sim$ $600 \mathrm{MHz}$ ) of the Fourier frequency $\omega, \mathrm{V}_{\mathrm{a} 1-\mathrm{b} 1}, \mathrm{~V}_{\mathrm{a} 1-} \sigma_{12}$, and $\mathrm{V} \sigma_{12-\mathrm{b} 1}$ remain steady values of about 2,1 , and 1 , except that there exist a narrow dip at the Fourier frequency $\omega=-1000 \mathrm{MHz}$ with the minimum values of about $1.11,0.55$, and 0.55 , respectively, which is due to the fact that at this particular Fourier frequency, the scattering and Stokes fields are resonant with the atomic transitions 2-3 and $1-3$.

The strong bipartite entanglement between the scattering field and generated Stokes field, as well as the atomic coherence can be well understood in terms of the interaction between the laser fields and atomic medium. As seen in Fig. 1a, the Stokes field $E_{1}$ is produced through FWM process, where every Stokes photon generation is accompanied by absorbing one scattering photon and one coupling photon and emitting one probe photon, and subsequent generation of atomic coherence excitation. Equivalently, as shown in Fig. 1b, the generated Stokes field can be regarded as the result of frequency down conversion process through mixing the scattering field with the atomic coherence prebuilt by the strong coupling and probe fields. Since a Stokes photon generation is always accompanied by annihilation of a scattering photon and creation of an atomic coherence excitation, strong tripartite entanglement can be established, which has the similar feature as the pump-signal-idler three-color entanglement produced by using an optical parametric oscillator ${ }^{4,5}$. In this respect, by using the EIT-induced atomic coherence between two different levels, a quantum frequency converter can be easily realized. The tripartite entanglement can also be seen clearly from Eqs. (5-6) and Eqs. (8-9). Due to the atomic coherence $\sigma_{12}^{(0)}$ preestablished via EIT, the output of the annihilation operators $a_{1}(L, \omega)$ and $b_{1}(L, \omega)$ are both a linear combination of the input of the operators $a_{1}(0, \omega)$ and $b_{1}(0, \omega)$ and Langevin noise terms, that is, the operators $a_{1}(L, \omega)$ and $b_{1}(L, \omega)$ are coupled together. If there is no atomic coherence $\sigma_{12}^{(0)}$, then the output operators $a_{1}(L, \omega)$ and $b_{1}(L, \omega)$ would have no mutual coupling of $a_{1}(0, \omega)$ and $b_{1}(0, \omega)$, and no correlation would exist between the scattering and Stokes fields. Also, as seen from Eq. (7), the first-order atomic operator $\sigma_{12}^{(1)}(z, \omega)$ is a linear combination of the output of the operators $a_{1}(L, \omega)$ and $b_{1}(L, \omega)$ and Langevin noise terms, which implies that the atomic coherence get entangled with both the scattering field and Stokes field. In this regard, the atomic coherence acts as a quantum beam splitter, similar to the polarizing beam splitter used in the traditional way. In principle, this idea can be generalized to the case with more scattering laser fields $\mathrm{E}_{\mathrm{m} 2}, \mathrm{E}_{\mathrm{m} 3}, \ldots . \mathrm{E}_{\mathrm{mN}}$ tuned to the vicinity of the transition $|1\rangle-|3\rangle$ and/or $|2\rangle-|3\rangle$ to mix with the same atomic coherence, then all of the scattering fields and generated Stokes/anti-Stokes fields would be entangled with the atomic coherence as well as with each other. This can be demonstrated by applying another field 


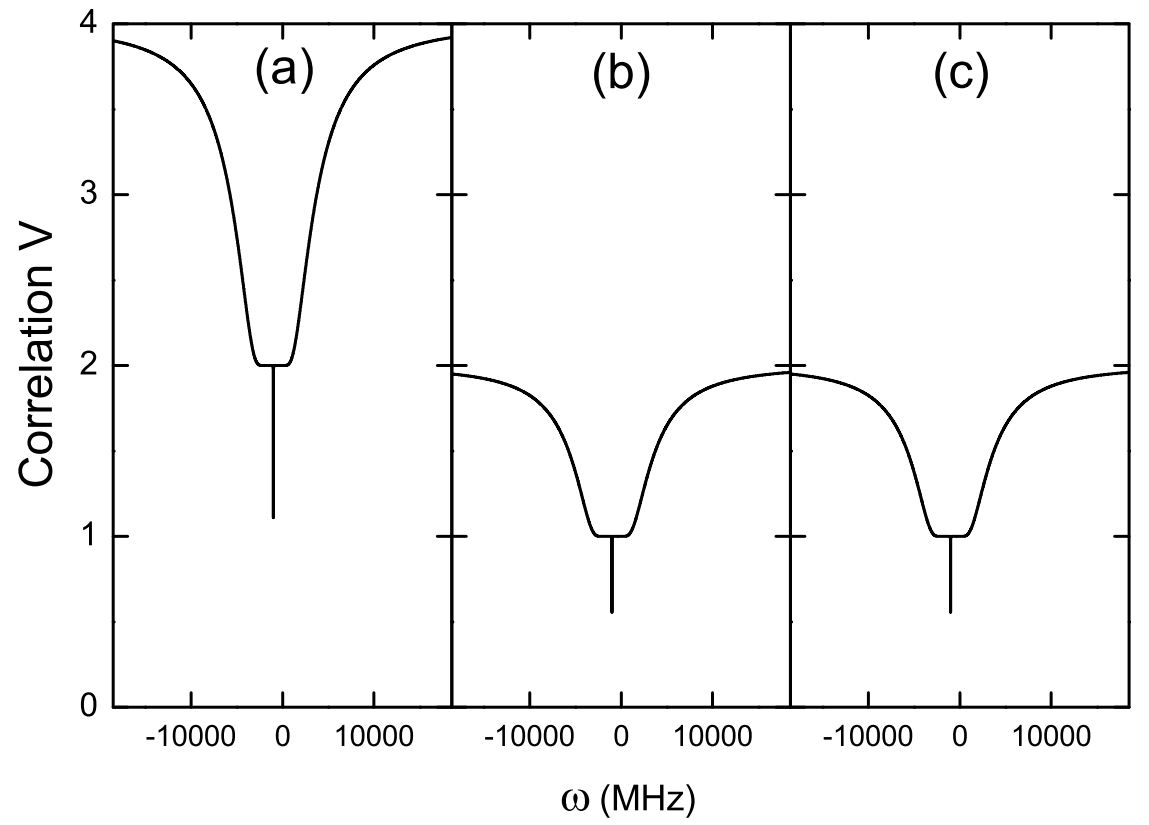

Figure 2 The evolution of correlations $\mathrm{V}_{\mathrm{a} 1-\mathrm{b} 1}(\mathrm{a}), \mathrm{V}_{\mathrm{a} 1-} \sigma_{12}(\mathrm{~b})$, and $\mathrm{V} \sigma_{12-\mathrm{b} 1}$ (c) as a function of the Fourier frequency $\omega$ with $L=0.06, r=1.0 \times 10^{-4}, n_{0}$

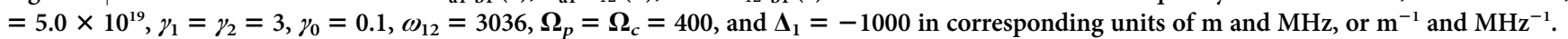

$\mathrm{E}_{\mathrm{m} 2}$ scattering off the atomic coherence to examine the entangled feature among the scattering fields $\mathrm{E}_{\mathrm{m} 1}$ and $\mathrm{E}_{\mathrm{m} 2}$, the generated Stokes and anti-Stokes fields $\mathrm{E}_{1}$ and $\mathrm{E}_{2}$, as well as the atomic coherence excitation.

Also, under the assumptions that the detunings $\Delta_{1}$ and $\Delta_{2}$ of the scattering fields are sufficiently large and the atomic coherence is strong enough, the evolution of the atomic operator $\sigma_{12}$ would include the terms from the interaction of the scattering field $\mathrm{E}_{\mathrm{m} 2}$ and the correspondingly generated anti-Stokes field $\mathrm{E}_{2}$ (described by annihilation operators $b_{2}$ and $a_{2}$, respectively) with the atomic medium, where the evolution of the operators $b_{2}$ and $a_{2}$ can be described by the same coupled propagation equation as the operators $a_{1}$ and $b_{1}$. In a similar way, by Fourier transforming the Heisenberg-Langevin equations and coupled propagation equations, we can get the output of the operators $a_{1}(L, \omega), a_{2}(L, \omega), b_{1}(L, \omega), b_{2}(L, \omega)$, and $\sigma_{12}(L, \omega)$.

Figure 3 shows the evolution of correlations $\mathrm{V}_{\mathrm{a} 1-\mathrm{a} 2}, \mathrm{~V}_{\mathrm{b} 1-\mathrm{b} 2}, \mathrm{~V}_{\mathrm{a} 1-} \sigma_{12}$, and $V \sigma_{12-b 1}$ as a function of the Fourier frequency $\omega$. It can be seen as well that, nearly in the whole range of the Fourier frequency $\omega$, the correlations are always less than 4 , which sufficiently demonstrates the generation of entanglement among the scattering fields $\mathrm{E}_{\mathrm{m} 1}$ and $\mathrm{E}_{\mathrm{m} 2}$, Stokes and anti-Stokes fields $\mathrm{E}_{1}$ and $\mathrm{E}_{2}$, as well as atomic coherence $\sigma_{12}$. The correlations $\mathrm{V}_{\mathrm{a} 1-} \sigma_{12}$, and $\mathrm{V} \sigma_{12 \text {-b1 }}$ have the similar features as the case without the scattering field $\mathrm{E}_{\mathrm{m} 2}$, which indicates that the scattering field $\mathrm{E}_{\mathrm{m} 2}$ has negligible influence on the bipartite entanglement. Also, the correlations $\mathrm{V}_{\mathrm{a} 1-\mathrm{a} 2}$ and $\mathrm{V}_{\mathrm{b} 1-\mathrm{b} 2}$ exhibit similar behaviors as $\mathrm{V}_{\mathrm{a} 1-\mathrm{b} 1}$, except that there exists two narrow dips with the minimum values of about 1.58 , which correspond to the two particular Fourier frequencies $\omega=1000 \mathrm{MHz}$ and $\omega=-1000 \mathrm{MHz}$, where the scattering fields $\mathrm{E}_{\mathrm{m} 2}$ and $\mathrm{E}_{\mathrm{m} 1}$ are resonant with the atomic transitions $1-3$ and $2-3$, respectively.

Based on the above analysis, it can be seen that the EIT-induced atomic coherence can be used to conveniently perform light-light, atom-atom, and atom-light entanglement distribution. For example, two EIT-based systems A and B similar to that shown in Fig. 1a can be established by Alice and Bob, respectively. Alice use a scattering field $\mathrm{E}_{\mathrm{Am} 1}$ mixing with the atomic coherence preproduced through EIT to generate a Stokes field $\mathrm{E}_{\mathrm{A} 1}$, and then send the scattering field and/or Stokes field through an optical fibre to Bob. Bob employs the scattering field $\mathrm{E}_{\mathrm{Am} 1}$ and/or Stokes field $\mathrm{E}_{\mathrm{A} 1}$ or another scattering field $\mathrm{E}_{\mathrm{Bm} 1}$ mixing with the atomic coherence precreated also through
EIT to generate a Stokes field $\mathrm{E}_{\mathrm{B} 1}$ and/or an anti-Stokes field $\mathrm{E}_{\mathrm{B} 2}$ or a Stokes (or an anti-Stokes) field $\mathrm{E}_{\mathrm{B} 3}$. Consequently, multipartite entanglement among the scattering field $\mathrm{E}_{\mathrm{Am} 1}$, Stokes field $\mathrm{E}_{\mathrm{A} 1}$, Stokes and/or anti-Stokes field $\mathrm{E}_{\mathrm{B} 1}, \mathrm{E}_{\mathrm{B} 21}$, and $\mathrm{E}_{\mathrm{B} 3}$, as well as the two atomic ensembles $\mathrm{A}$ and $\mathrm{B}$, can be established. Therefore, by sequentially performing such operations, multipartite entanglement distribution over long distance can be easily achieved with only coherent light fields.

The robustness of entanglement is a key issue for practical quantum applications. In the present $\Lambda$-type atomic system, it critically depends on the coherence decay rate of the lower doublet. This is clearly demonstrated in Fig. 4 by examining the influence of the coherence decay rate $\gamma_{0}$ on the correlations $\mathrm{V}_{\mathrm{a} 1-\mathrm{b} 1}, \mathrm{~V}_{\mathrm{a} 1-} \sigma_{12}$, and $\mathrm{V} \sigma_{12-\mathrm{b} 1}$ at zero Fourier frequency. $\mathrm{V}_{\mathrm{a} 1-\mathrm{b} 1}, \mathrm{~V}_{\mathrm{a} 1-} \sigma_{12}$, and $\mathrm{V} \sigma_{12-\mathrm{b} 1}$ with the initial values of about 2,1 , and 1 , respectively, would increase with the increase of the coherence decay rate $\gamma_{0}$, which means the degree of entanglement would be weakened. When the coherence decay rate $\gamma_{0}$ grows large enough, $\mathrm{V}_{\mathrm{a} 1 \text {-b1 }}$ nearly becomes equal to 4 , whereas $\mathrm{V}_{\mathrm{a} 1-} \sigma_{12}$ and $\mathrm{V} \sigma_{12 \text {-b1 }}$ approximately approach 2 . In the realistic hot atomic ensemble, the source of decoherence associated to the ground states is mainly due to the finite interaction time between atoms and light. According to the experimental conditions in Refs. $[17,28,33]$ with the beam radius $r$ being of about 300 um and cell temperature of about $400 \mathrm{~K}$, it can be inferred that the dephasing time of the atomic spin coherence is on the order of microsecond; correspondingly, the coherence decay rate of the lower doublet is about several MHz. As seen from Fig. 4, this low relaxation rate has a negligible impact on the entanglement. In order to conveniently and successfully measure the entanglement, the experimental setup with a square-box pattern for the laser beams having spatial separation and nearly complete cancellation of Doppler broadening done in Ref. [28], and the dual-homodyne detection method in Ref. [17] together with the photodetector (S3883 Si pin photodiodes) having quantum efficiency of about $96 \%$ used in Ref. [33], can be employed. Moreover, it is well known that, in the $\Lambda$-type atomic system, the correlation time of the entangled fields and the storage time of the quantum memory are determined by the coherence decay time of the atomic lower doublet, which, in practice, can be long $(\sim m s$ or even $\sim s^{24-26,34,35}$ ). Therefore, this EIT-based multi- $\Lambda$-type atomic system can be a potential candidate for a quantum repeater, through which 


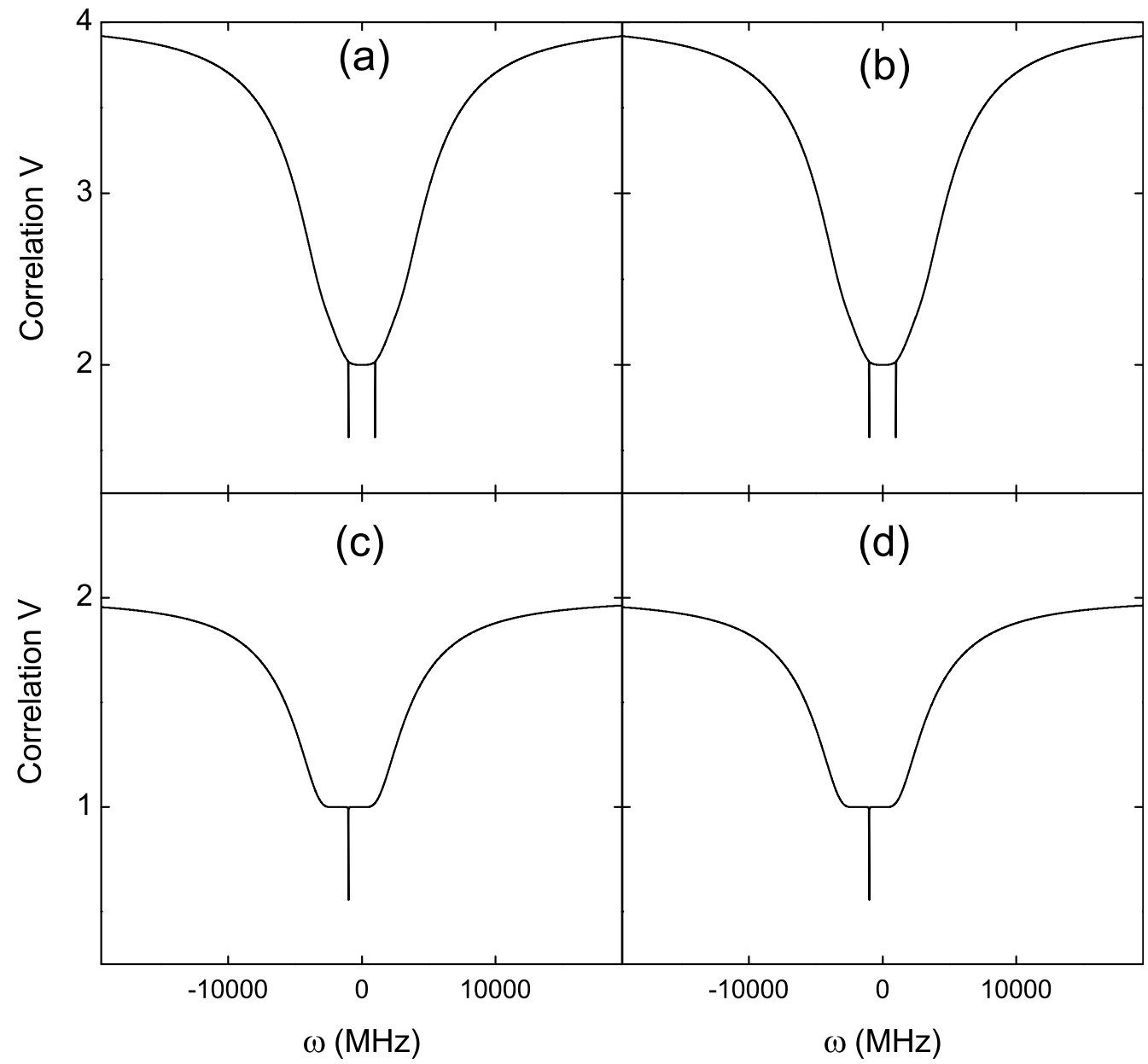

Figure $3 \mid$ The evolution of correlations $\mathrm{V}_{\mathrm{a} 1-\mathrm{a} 2}(\mathrm{a}), \mathrm{V}_{\mathrm{b} 1-\mathrm{b} 2}(\mathrm{~b}), \mathrm{V}_{\mathrm{a} 1-} \sigma_{12}(\mathrm{c})$, and $\mathrm{V} \sigma_{12-\mathrm{b} 1}(\mathrm{~d})$ as a function of the Fourier frequency $\omega$ with $\Delta_{2}=-\Delta_{1}=$ 1000 , and the other parameters are the same as those in Fig. 2.

quantum state distribution over long distance can be achieved via successive entanglement exchange and distribution. In addition, as depicted in Fig. 5, bipartite entanglements between the scattering fields, Stokes and anti-Stokes fields, as well as the atomic coherence excitation are independent of the intensity $|\alpha|^{2}$ of the scattering fields. So we can use bright enough beams to carry quantum information as long as the detunings $\Delta_{1}$ and $\Delta_{2}$ of the scattering fields are sufficiently large and the atomic coherence is strong enough.

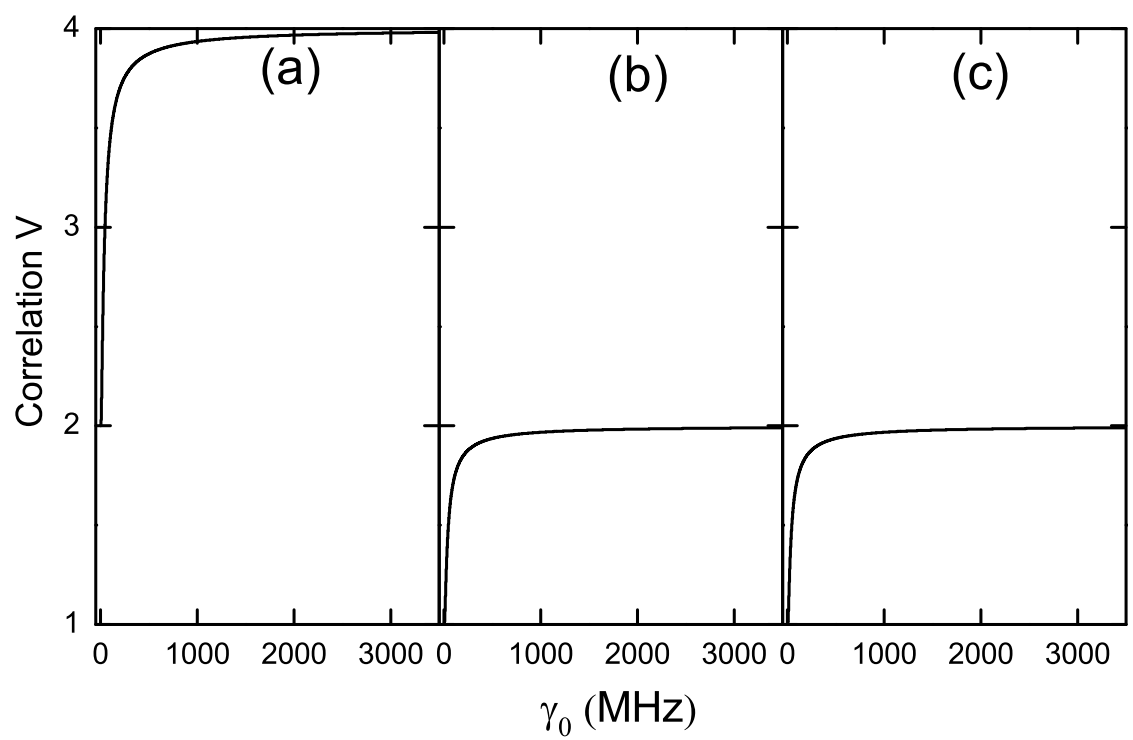

Figure $4 \mid$ The dependence of correlations $\mathrm{V}_{\mathrm{a1}-\mathrm{b} 1}(\mathrm{a}), \mathrm{V}_{\mathrm{a} 1-} \sigma_{12}(\mathrm{~b})$, and $\mathrm{V} \sigma_{12-\mathrm{b} 1}(\mathrm{c})$ at zero Fourier frequency on the coherence decay rate $\gamma_{0}$, and the other parameters are the same as those in Fig. 2. 


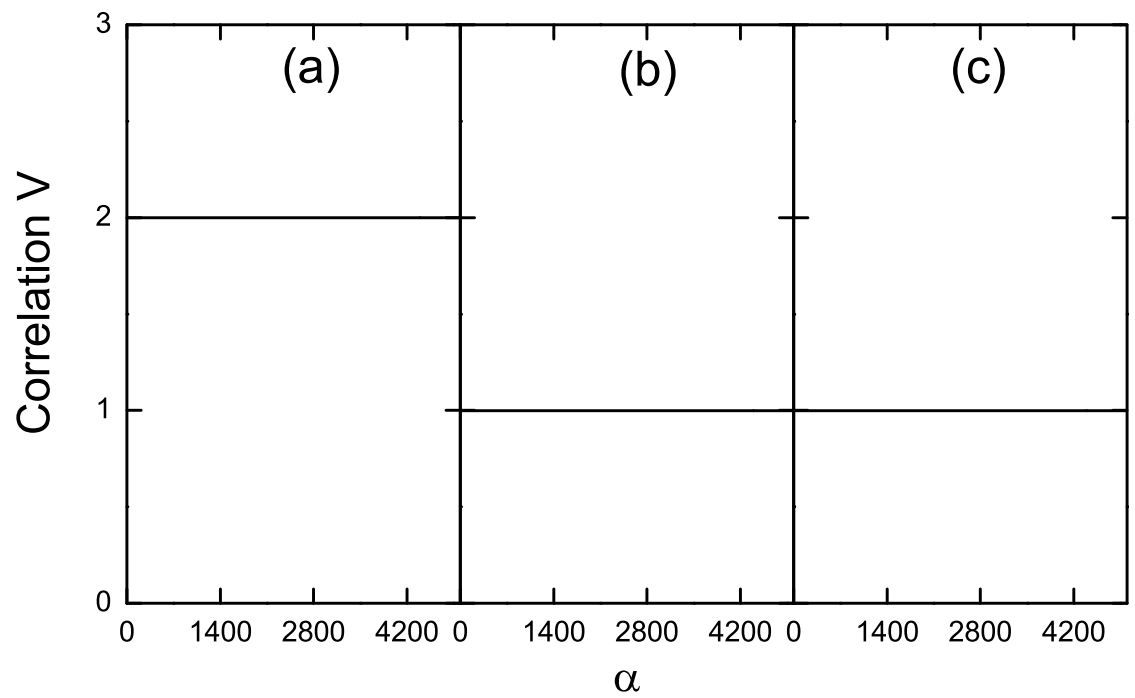

Figure 5 The dependence of correlations $\mathrm{V}_{\mathrm{a} 1-\mathrm{bl}}(\mathrm{a}), \mathrm{V}_{\mathrm{a} 1-} \sigma_{12}(\mathrm{~b})$, and $\mathrm{V} \sigma_{12-\mathrm{bl}}(\mathrm{c})$ at zero Fourier frequency on $\alpha$, and the other parameters are the same as those in Fig. 2.

\section{Discussion}

It is worth noting that the present scheme for quantum repeater is quite distinct as compared to that proposed by Yuan ${ }^{24}$. In Ref. [24], the Stokes or anti-Stokes field is produced through spontaneous Raman scattering and would emit in all directions, whereas in the present scheme, the generated Stokes or anti-Stokes field would propagate along a particular direction determined by the phasematching condition for $\mathrm{FWM}^{28}$ and the photon production efficiency is much higher; also, nonclassical input light fields and interferometric stabilization over long distance are required for entanglement swapping and the entanglement is created in a post-selection way, whereas in the present case, multipartite entanglement exchange and distribution can be conveniently and efficiently realized in a deterministic way via atomic coherence by using only coherent input light fields and can avoid the difficulty in keeping interferometric stabilization, which would greatly simplify the practical implementation.

In conclusion, we have presented an efficient and convenient scheme for achieving a universal quantum information processor via atomic coherence by employing nondegenerate four-wave mixing processes in the multi- $\Lambda$-type atomic system, which has full functions of quantum beam splitter, quantum frequency converter, quantum entangler, and quantum repeater. By using the QIP, quantum state exchange, distribution, and manipulation between light and light, matter and matter, as well as light and matter can be efficiently and flexibly achieved deterministically with only coherent light fields. This method would bring great facility in realistic quantum information processing protocols, and may find potential applications in scalable quantum communication and quantum networks.

1. Bouweester, D., Ekert, A. \& Zeilinger, A. The Physics of Quantum Information (Springer-Verlag, Berlin, 2000).

2. Nielsen, M. A. \& Chuang, I. L. Quantum Computation and Quantum Information (Cambridge University Press, Cambridge, 2000).

3. Kimble, H. J. The quantum internet. Nature 453, 1023-1030 (2008).

4. Villar, A. S., Martinelli, M., Fabre, C. \& Nussenzveig, P. Direct production of tripartite pump-signal-idler entanglement in the above-threshold optical parametric oscillator. Phys. Rev. Lett. 97, 140504 (2006).

5. Coelho, A. S. et al. Three-color entanglement. Science 326, 823 (2009).

6. Cassemiro, K. N. \& Villar, A. S. Scalable continuous-variable entanglement of light beams produced by optical parametric oscillators. Phys. Rev. A 77, 022311 (2008).

7. Olsen, M. K. \& Bradley, A. S. Asymmetric polychromatic tripartite entanglement from interlinked $\chi^{(2)}$ parametric interactions. Phys. Rev. A 74, 063809 (2006).

8. Pfister, O. et al. Multipartite continuous-variable entanglement from concurrent nonlinearities. Phys. Rev. A 70, 020302 (2004).
9. Arimondo, E. Coherent population trapping in laser spectroscopy. Prog. Opt. 35, 257 (1996).

10. Harris, S. E. Electromagnetically induced transparency. Phys. Today 50, 36-42 (1997).

11. Fleischhauer, M., Imamoglu, A. \& Marangos, J. P. Electromagnetically induced transparency: Optics in coherent media. Rev. Mod. Phys. 77, 633 (2005).

12. Duan, L. M., Lukin, M. D., Cirac, J. I. \& Zoller, P. Long-distance quantum communication with atomic ensembles and linear optics. Nature 414, 413 (2001).

13. Van Der Wal, C. H. et al. Atomic memory for correlated photon states. Science 301, 196 (2003).

14. Kuzmich, A. et al. Generation of nonclassical photon pairs for scalable quantum communication with atomic ensembles. Nature 423, 731 (2003).

15. Lukin, M. D., Hemmer, P. R. \& Scully, M. O. Resonant nonlinear optics in phasecoherent media. Adv. At. Mol. Opt. Phys. 42, 347 (2000).

16. Balic, V. et al. Generation of paired photons with controllable waveforms. Phys. Rev. Lett. 94, 183601 (2005).

17. Boyer, V., Marino, A. M., Pooser, R. C. \& Lett, P. D. Entangled images from fourwave mixing. Science 321, 544-547 (2008).

18. Yang, X. H., Zhou, Y. Y. \& Xiao, M. Generation of multipartite continuousvariable entanglement via atomic spin wave. Phys. Rev. A 85, 052307 (2012).

19. Yang, X. H., Zhou, Y. Y. \& Xiao, M. Entangler via electromagnetically induced transparency with an atomic ensemble. Sci. Rep. 3, 3479 (2013).

20. Yang, X. H. et al. Generation of multipartite continuous-variable entanglement via atomic spin wave: Heisenberg-Langevin approach. Opt. Exp. 22, 12563 (2014).

21. Duan, L. M., Cirac, J. I., Zoller, P. \& Polzik, E. S. Quantum communication between atomic ensembles using coherent light. Phys. Rev. Lett. 85, 5643 (2000).

22. Julsgaard, B., Kozhekin, A. \& Polzik, E. S. Experimental long-lived entanglement of two macroscopic objects. Nature 413, 400 (2001).

23. Choi, K. S., Deng, H., Laurat, J. \& Kimble, H. J. Mapping photonic entanglement into and out of a quantum memory. Nature 452, 67 (2008).

24. Yuan, Z. S. et al. Experimental demonstration of a BDCZ quantum repeater node. Nature 454, 1098 (2008).

25. Sangouard, N., Simon, C., de Riedmatten, H. \& Gisin, N. Quantum repeaters based on atomic ensembles and linear optics. Rev. Mod. Phys. 83, 33 (2011).

26. Pan, J. W. et al. Multiphoton entanglement and interferometry. Rev. Mod. Phys. 84, 777 (2012).

27. Briegel, H. J., Dür, W., Cirac, J. I. \& Zoller, P. Quantum Repeaters: The role of imperfect local operations in quantum communication. Phys. Rev. Lett. 81, 5932 (1998).

28. Yang, X. H., Sheng, J. T., Khadka, U. \& Xiao, M. Generation of correlated and anticorrelated multiple fields via atomic spin coherence. Phys. Rev. A 85, 013824 (2012).

29. Fleischhauer, M. \& Richter, T. Pulse matching and correlation of phase fluctuations in $\Lambda$ systems. Phys. Rev. A 51, 2430 (1995).

30. Kolchin, P. Electromagnetically-induced-transparency-based paired photon generation. Phys. Rev. A 75, 033814 (2007).

31. Glorieux, Q. et al. Double- $\Lambda$ microscopic model for entangled light generation by four-wave mixing. Phys. Rev. A 82, 033819 (2010).

32. Duan, L. M., Giedke, G., Cirac, J. I. \& Zoller, P. Inseparability criterion for continuous variable systems. Phys. Rev. Lett. 84, 2722-2725 (2000).

33. Qin, Z. Z. et al. Experimental generation of multiple quantum correlated beams from hot Rubidium vapor. Phys. Rev. Lett. 113, 023602 (2014). 
34. Zhang, R., Garner, S. \& Hau, L. V. Creation of long-term coherent optical memory via controlled nonlinear interactions in Bose-Einstein condensates. Phys. Rev. Lett. 103, 233602 (2009).

35. Schnorrberger, U. et al. Electromagnetically induced transparency and light storage in an atomic mott insulator. Phys. Rev. Lett. 103, 033003 (2009).

\section{Acknowledgments}

This work is supported the National Natural Science Foundation of China (Nos. 11274225 10974132, and 50932003), Key Basic Research Program of Shanghai Municipal Science and Technology Commission (No. 14JC1402100), and Shanghai Natural Science Foundation (14ZR1415400).

\section{Author contributions}

X.Y. and S.Z. conceived the idea for this project. X.Y. and B.X. performed the calculations and prepared figures 1-5. X.Y. and J.Z. wrote the main manuscript text. All authors reviewed the manuscript.

\section{Additional information}

Competing financial interests: The authors declare no competing financial interests.

How to cite this article: Yang, X., Xue, B., Zhang, J. \& Zhu, S. A universal quantum information processor for scalable quantum communication and networks. Sci. Rep. 4, 6629; DOI:10.1038/srep06629 (2014).

This work is licensed under a Creative Commons Attribution-NonCommercialShareAlike 4.0 International License. The images or other third party material in this article are included in the article's Creative Commons license, unless indicated otherwise in the credit line; if the material is not included under the Creative Commons license, users will need to obtain permission from the license holder in order to reproduce the material. To view a copy of this license, visit http:// creativecommons.org/licenses/by-nc-sa/4.0/ 